\title{
Stochastic signaling in the presence of channel state information uncertainty
}

\author{
Cagri Goken ${ }^{\mathrm{a}}$, Sinan Gezici ${ }^{\mathrm{b}, *}$, Orhan Arikan ${ }^{\mathrm{b}}$ \\ a Department of Electrical Engineering, Princeton University, Princeton, NJ 08544, USA \\ ${ }^{\mathrm{b}}$ Department of Electrical and Electronics Engineering, Bilkent University, Ankara 06800, Turkey
}

\section{A R T I C L E I N F O}

Article history:

Available online 22 October 2012

\section{Keywords:}

Probability of error

Stochastic signaling

Channel state information

Minimax

\begin{abstract}
A B S T R A C T
In this paper, stochastic signaling is studied for power-constrained scalar valued binary communications systems in the presence of uncertainties in channel state information (CSI). First, stochastic signaling based on the available imperfect channel coefficient at the transmitter is analyzed, and it is shown that optimal signals can be represented by a randomization between at most two distinct signal levels for each symbol. Then, performance of stochastic signaling and conventional deterministic signaling is compared for this scenario, and sufficient conditions are derived for improvability and nonimprovability of deterministic signaling via stochastic signaling in the presence of CSI uncertainty. Furthermore, under CSI uncertainty, two different stochastic signaling strategies, namely, robust stochastic signaling and stochastic signaling with averaging, are proposed. For the robust stochastic signaling problem, sufficient conditions are derived for reducing the problem to a simpler form. It is shown that the optimal signal for each symbol can be expressed as a randomization between at most two distinct signal values for stochastic signaling with averaging, as well as for robust stochastic signaling under certain conditions. Finally, two numerical examples are presented to explore the theoretical results.
\end{abstract}

(c) 2012 Elsevier Inc. All rights reserved.

\section{Introduction}

In binary communications systems over zero-mean additive white Gaussian noise (AWGN) channels and under average power constraints in the form of $\mathrm{E}\left\{\left|S_{i}\right|^{2}\right\} \leqslant A$ for $i=0,1$, the average probability of error is minimized when deterministic antipodal signals $\left(S_{0}=-S_{1}\right)$ are used at the power limit $\left(\left|S_{0}\right|^{2}=\left|S_{1}\right|^{2}=A\right)$ and a maximum a posteriori probability (MAP) decision rule is used at the receiver [2]. Also, for vector observations, selecting the deterministic signals along the eigenvector of the covariance matrix of the Gaussian noise corresponding to the minimum eigenvalue minimizes the average probability of error [2]. In [3], optimal binary communications over AWGN channels are investigated for nonequal prior probabilities under an average energy per bit constraint, and it is shown that the optimal signaling scheme is on-off keying (OOK) for coherent detection when the signals have nonnegative correlation (also for envelope detection for arbitrary signal correlation).

\footnotetext{
th This research was supported in part by the National Young Researchers Career Development Programme (project No. 110E245) of the Scientific and Technological Research Council of Turkey (TUBITAK). Part of this work was presented at the IEEE Global Communications Conference (GLOBECOM 2011), Houston, Texas, USA [1].

* Corresponding author. Fax: +90 3122664192.

E-mail addresses: cgoken@princeton.edu (C. Goken), gezici@ee.bilkent.edu.tr (S. Gezici), oarikan@ee.bilkent.edu.tr (O. Arikan).
}

In [4], the convexity properties of the average probability of error in terms of signal and noise power are investigated for binaryvalued scalar signals over additive noise channels under an average power constraint. First, it is shown that randomization of signal values (or, stochastic signaling) cannot improve the error performance of a maximum likelihood (ML) detector at the receiver when the average probability of error is a convex nonincreasing function of the signal power. Then, the problem of maximizing the average probability of error is studied for an average powerconstrained jammer, and it is shown that the optimal solution can be obtained when the jammer randomizes its power between at most two power levels. In [5], the results in [4] are generalized by exploring the convexity properties of the error rates for constellations with arbitrary shape, order, and dimensionality for an ML detector in AWGN with no fading and with frequency-flat slowly fading channels. Also, the investigations in [4] for optimum power/time sharing for a jammer to maximize the average probability of error and the optimum transmission strategy to minimize the average probability of error are extended to arbitrary multidimensional constellations for AWGN channels [5].

While the optimal signaling structures are well-known in the presence of Gaussian noise (e.g., [2,5]), the noise can have significantly different probability distribution from the Gaussian distribution in some cases due to effects such as interference and jamming $[4,6,7]$. When the noise is non-Gaussian, the results in $[4,8-10]$ imply that signal randomization can provide 
performance improvements in terms of average probability of error reduction compared to the conventional deterministic signaling. In [10], the design of stochastic signals for each symbol is studied, and the improvements that can be achieved via this stochastic signaling approach are investigated. For a given decision rule (detector) at the receiver, optimal stochastic signals are obtained under second and fourth moment constraints, and it is shown that an optimal stochastic signal can be represented by a randomization among at most three distinct signal values for each symbol [10]. Also, sufficient conditions are obtained to specify whether stochastic signaling provides improvements over deterministic signaling. In [11], stochastic signaling is studied under an average power constraint in the form of $\sum_{i=1}^{2} \pi_{i} \mathrm{E}\left\{\left|S_{i}\right|^{2}\right\} \leqslant A$, where $S_{i}$ denotes the $i$ th signal and $\pi_{i}$ denotes the prior probability of symbol $i$. Sufficient conditions are presented to determine performance improvements. Also, [12] investigates the joint design of the optimal stochastic signals and the detector, and proves that the optimal solution involves randomization between at most two signal values and the use of the corresponding MAP detector. In addition, in [13], randomization between two deterministic signal pairs and the corresponding MAP decision rules is studied, and significant performance improvements via power randomization are observed. Finally, in some studies such as [14-19], time-varying or random signal constellations are employed in order to improve error performance or to achieve diversity.

Although optimal stochastic signaling for power-constrained communications systems has been studied in [10-12], no studies have considered the effects of imperfect channel state information (CSI) on the performance of stochastic signaling and the design of stochastic signals under CSI uncertainty. In this study, we first investigate stochastic signaling based on imperfect CSI (considering generic noise probability distributions and detector structures), and analyze the effects of imperfect CSI on stochastic signaling. After the formulation of stochastic signaling under CSI uncertainty, we state that an optimal stochastic signal involves randomization between at most two distinct signal levels. Then, we derive sufficient conditions to specify when the use of stochastic signaling can or cannot provide improvements over conventional signaling in the presence of imperfect CSI.

Secondly, we propose two different methods, namely, robust stochastic signaling and stochastic signaling with averaging, for designing stochastic signals under CSI uncertainty. In robust stochastic signaling, signals are designed for the worst-case channel coefficients, and the optimal signaling problem is formulated as a minimax problem $[2,20]$. Then, sufficient conditions under which the generic minimax problem is equivalent to designing signals for the smallest possible magnitude of the channel coefficient are obtained. In the stochastic signaling with averaging approach, the transmitter assumes a probability distribution for the channel coefficient, and stochastic signals are designed by averaging over different channel coefficient values based on that probability distribution. It is shown that optimal signals obtained after this averaging method and those for the equivalent form of the robust signaling method can be represented by randomization between at most two distinct signal levels for each symbol. Solutions for the optimization problems can be calculated by using global optimization techniques such as particle swarm optimization (PSO) [21], or convex relaxation approaches can be employed as in [10,22-25]. Finally, we perform simulations and present two numerical examples to illustrate the theoretical results.

\section{System model and motivation}

Consider a binary communications system with scalar observations $[4,26]$, in which the channel effect is modeled by a mul- tiplicative term as in flat-fading channels [27], and the received signal is given by

$Y=\alpha S_{i}+N, \quad i \in\{0,1\}$,

where $S_{0}$ and $S_{1}$ denote the transmitted signal values for symbol 0 and symbol 1 , respectively, $\alpha$ is the channel coefficient, and $N$ is the noise component that is independent of $S_{i}$ and $\alpha$. In addition, the prior probabilities of the symbols, which are denoted by $\pi_{0}$ and $\pi_{1}$, are supposed to be known

In (1), the noise term $N$ is modeled to have an arbitrary probability distribution considering that it can include the combined effects of thermal noise, interference, and jamming. Hence, the probability distribution of the noise component is not necessarily Gaussian [6].

A generic decision rule is considered at the receiver to determine the symbol in (1). For a given observation $Y=y$, the decision rule $\phi(y)$ is expressed as

$\phi(y)= \begin{cases}0, & y \in \Gamma_{0}, \\ 1, & y \in \Gamma_{1},\end{cases}$

where $\Gamma_{0}$ and $\Gamma_{1}$ are the decision regions for symbol 0 and symbol 1 , respectively [2].

The aim is to design signals $S_{0}$ and $S_{1}$ in (1) in order to minimize the average probability of error for a given decision rule, which is calculated as

$\mathrm{P}_{\mathrm{avg}}=\pi_{0} \mathrm{P}_{0}\left(\Gamma_{1}\right)+\pi_{1} \mathrm{P}_{1}\left(\Gamma_{0}\right)$,

with $\mathrm{P}_{i}\left(\Gamma_{j}\right)$ denoting the probability of selecting symbol $j$ when symbol $i$ is transmitted. In practical systems, there exists an average power constraint on each of the signals, which can be expressed as [2]

$\mathrm{E}\left\{\left|S_{i}\right|^{2}\right\} \leqslant A$,

for $i=0,1$, where $A$ is the average power limit. Therefore, in the stochastic signaling approach, the aim becomes the calculation of the optimal probability density functions (PDFs) for signals $S_{0}$ and $S_{1}$ that minimize the average probability of error in (3) under the average power constraint in (4) [10]. In other words, in the stochastic signal design, the signals at the transmitter are modeled as random variables and the optimal PDFs of these random variables are obtained.

Unlike stochastic signaling, in the conventional signal design, $S_{0}$ and $S_{1}$ are modeled as deterministic signals and set to $S_{0}=-\sqrt{A}$ and $S_{1}=\sqrt{A}[2,27]$. Then, the average probability of error in (3) becomes

$\mathrm{P}_{\text {conv }}=\pi_{0} \int_{\Gamma_{1}} p_{N}(y+\alpha \sqrt{A}) d y+\pi_{1} \int_{\Gamma_{0}} p_{N}(y-\alpha \sqrt{A}) d y$,

where $p_{N}(\cdot)$ is the PDF of the noise in (1).

As investigated in [10-12], stochastic signaling results in lower average probabilities of error than conventional deterministic signaling in some cases in the presence of non-Gaussian noise. However, the common assumption in the previous studies is that the channel coefficient $\alpha$ in (1) is known perfectly at the transmitter, i.e., the CSI is available at the transmitter. In practice, the transmitter can obtain CSI via feedback from the receiver, or by utilizing the reciprocity of forward and reverse links under time-division duplexing [28]. In both scenarios, it is realistic to model the CSI at the transmitter to include certain errors/uncertainties. Therefore, the main motivation behind this study is to investigate stochastic signaling under imperfect CSI; that is, to evaluate the performance of stochastic signaling in practical scenarios and to develop different design methods for stochastic signaling under CSI uncertainty. In the next section, the effects of CSI uncertainties on the performance of stochastic signaling are examined. 
Remark 1. The use of stochastic signaling can provide performance improvements for communications systems that operate in the presence of non-Gaussian noise [10]. For example, stochastic signaling can be employed for the downlink of a multiuser direct-sequence spread-spectrum (DSSS) system, in which Gaussian mixture noise is observed at the receiver of each user due to the presence of multiple-access interference and Gaussian background noise [29]. For practical implementation, the transmitter needs to know the channel condition for each user, which can be sent via feedback to the transmitter. In addition, stochastic signaling can be regarded as a signal randomization for each information symbol [10], which can, for example, be implemented via time sharing (i.e., sending different signal values for certain durations of time). In that case, channel coefficients should be constant during the randomization operation; hence, slow fading channels are well-suited for stochastic signaling.

\section{Effects of channel uncertainties on the stochastic signaling}

\subsection{Stochastic signaling with imperfect channel coefficients}

In the stochastic signaling approach, signals $S_{0}$ and $S_{1}$ in (1) are modeled as random variables and their optimal PDFs are searched for. Let $p_{S_{0}}(\cdot)$ and $p_{S_{1}}(\cdot)$ represent the PDFs of $S_{0}$ and $S_{1}$, respectively. Also define $\hat{S}_{0} \triangleq \alpha S_{0}$ and $\hat{S}_{1} \triangleq \alpha S_{1}$, and denote their PDFs as $p_{\hat{S}_{0}}(\cdot)$ and $p_{\hat{S}_{1}}(\cdot)$, respectively. Then, from (3), the average probability of error for the decision rule in (2) can be obtained as

$\mathrm{P}_{\text {stoc }}=\sum_{i=0}^{1} \pi_{i} \int_{-\infty}^{\infty} p_{\hat{S}_{i}}(t) \int_{\Gamma_{1-i}} p_{N}(y-t) d y d t$.

Since $p_{\hat{S}_{i}}(t)$ can be obtained as $p_{\hat{S}_{i}}(t)=(1 /|\alpha|) p_{S_{i}}(t / \alpha)$ for $i=$ $0,1,(6)$ can be expressed, after a change of variable $(t=\alpha x)$, as

$\mathrm{P}_{\text {stoc }}=\sum_{i=0}^{1} \pi_{i} \int_{-\infty}^{\infty} p_{S_{i}}(x) \int_{\Gamma_{1-i}} p_{N}(y-\alpha x) d y d x$.

Since imperfect CSI is considered in this study, the transmitter has a distorted version of the correct channel coefficient $\alpha$. Let $\hat{\alpha}$ denote this distorted (noisy) channel coefficient at the transmitter. In this section, it is assumed that the transmitter uses $\hat{\alpha}$ in the design of stochastic signals. Then, the stochastic signal design problem can be expressed as

$$
\begin{aligned}
& \min _{p_{S_{0}}, p_{S_{1}}} \sum_{i=0}^{1} \pi_{i} \int_{-\infty}^{\infty} p_{S_{i}}(x) \int_{\Gamma_{1-i}} p_{N}(y-\hat{\alpha} x) d y d x \\
& \text { subject to } \mathrm{E}\left\{\left|S_{i}\right|^{2}\right\} \leqslant A, i=0,1 .
\end{aligned}
$$

Note that there are also implicit constraints in the optimization problem in (8) because $p_{S_{0}}(\cdot)$ and $p_{S_{1}}(\cdot)$ need to satisfy the conditions to be valid PDFs. Similarly to [10], this optimization problem can be expressed as two separate optimization problems for $S_{0}$ and $S_{1}$. Namely, the optimal signal PDF for symbol 1 can be obtained from the solution of the following optimization problem:

$\min _{p_{S_{1}}} \int_{-\infty}^{\infty} p_{S_{1}}(x) \int_{\Gamma_{0}} p_{N}(y-\hat{\alpha} x) d y d x$ subject to $\mathrm{E}\left\{\left|S_{1}\right|^{2}\right\} \leqslant A$.

If $G(x, k)$ is defined as

$G(x, k) \triangleq \int_{\Gamma_{0}} p_{N}(y-k x) d y$,
(9) can also be written as

$\min _{p_{S_{1}}} \mathrm{E}\left\{G\left(S_{1}, \hat{\alpha}\right)\right\} \quad$ subject to $\mathrm{E}\left\{\left|S_{1}\right|^{2}\right\} \leqslant A$,

where the expectations are taken over $S_{1}$. Note that $G\left(S_{1}, \hat{\alpha}\right)$ is only a function of $S_{1}$ for a given value of $\hat{\alpha}$. In some previous studies, such as [10], [13], and [30], the optimization problems in the same form as that in (11) have been explored thoroughly. If $G\left(S_{1}, \hat{\alpha}\right)$ in (11) is a continuous function of $S_{1}$, and $S_{1}$ takes values in $[-\gamma, \gamma]$ for some finite positive $\gamma$, then the optimal solution of (11) can be represented by a randomization between at most two distinct signal levels as a result of Carathéodory's theorem [31]. Hence, the optimal signal PDF for $S_{1}$ can be expressed as

$p_{S_{1}}(s)=\lambda_{1} \delta\left(s-s_{11}\right)+\left(1-\lambda_{1}\right) \delta\left(s-s_{12}\right), \quad \lambda_{1} \in[0,1]$.

A similar optimization problem can also be formulated for $S_{0}$. After obtaining the optimal signal PDFs for $S_{0}$ and $S_{1}$, the corresponding average probability of error can be calculated. Since the optimization problems are similar for $S_{0}$ and $S_{1}$, we focus on the design of $S_{1}$ in the remainder of this section.

\subsection{Stochastic signaling versus conventional signaling}

It is known that, in the presence of perfect CSI at the transmitter, conventional signaling, which sets $S_{1}=\sqrt{A}$ [that is, $p_{S_{1}}(x)=$ $\delta(x-\sqrt{A})$ ], can or cannot be optimal under certain sufficient conditions as discussed in [10]. In this section, we explore the conditions under which the use of stochastic signaling instead of deterministic signaling can or cannot result in improved average probability of error performance in the presence of imperfect CSI.

In the presence of imperfect CSI, let the transmitter have the channel coefficient information as $\hat{\alpha}$. Then, the transmitter obtains the optimal stochastic signal $S_{1}$ from (11). Let $p_{S_{1}}^{\hat{\alpha}}(\cdot)$ denote the solution of (11) for a given value of $\hat{\alpha}$. Then, the corresponding conditional probability of error for symbol 1 is given by

$\mathrm{P}_{\mathrm{e}}^{\hat{\alpha}}=\int_{-\infty}^{\infty} p_{S_{1}}^{\hat{\alpha}}(x) G(x, \alpha) d x$

where $G(x, \alpha)$ is as defined in (10). Note that $G(x, \alpha)$ specifies the probability of choosing symbol 0 for a given signal value $x$ for symbol 1 when the channel coefficient is equal to $\alpha$. Therefore, when the stochastic signal for symbol 1 is specified by the PDF $p_{S_{1}}^{\hat{\alpha}}(x)$, the corresponding conditional probability of error for symbol 1 is obtained as in (13).

Suppose that $\hat{\alpha}$ can be modeled as a random variable with a generic PDF $p_{\hat{\alpha}}(\cdot)$. In order to improve the performance of conventional signaling for symbol 1 via stochastic signaling, we need to have $\mathrm{P}_{\mathrm{e}}<G(\sqrt{A}, \alpha)$, where $G(\sqrt{A}, \alpha)$ is the conditional probability of error for conventional signaling, i.e., for $S_{1}=\sqrt{A}$ (see (5) and (10)), and $\mathrm{P}_{\mathrm{e}}$ is the average conditional probability of error for stochastic signaling based on imperfect CSI, which can be calculated as

$\mathrm{P}_{\mathrm{e}}=\int_{-\infty}^{\infty} p_{\hat{\alpha}}(a) \mathrm{P}_{\mathrm{e}}^{a} d a$

with $\mathrm{P}_{\mathrm{e}}^{a}$ being given by (13).

In order to derive sufficient conditions for the improvability and nonimprovability of conventional signaling via stochastic signaling, assume that the channel coefficient information at the transmitter is specified as $\hat{\alpha}=\alpha+\eta$, where $\eta$ is a zero-mean Gaussian noise with standard deviation $\varepsilon$; that is, $\eta \sim \mathcal{N}\left(0, \varepsilon^{2}\right)$. Although 
the Gaussian error model is employed for the convenience of the analysis, the results are valid also for non-Gaussian error models, as will be discussed at the end of this section. In addition, it is assumed that $\alpha$ is a positive number without loss of generality. ${ }^{1}$ Then, the following proposition presents sufficient conditions on the improvability and nonimprovability of conventional signaling via stochastic signaling.

Proposition 1. Assume that $G(x, k)$ in (10) and $\mathrm{P}_{\mathrm{e}}^{\hat{\alpha}}$ in (13) have the following properties:

- $G(x, k)$ is a strictly decreasing function of $x$ for any fixed positive $k$, and $G(x, k)=1-G(-x, k)$.

- There exist $\kappa_{1}, \kappa_{2}, \gamma_{t h}, \theta_{t h}$, and $\beta_{t h}$ such that $\mathrm{P}_{\mathrm{e}}^{\hat{\alpha}}<\kappa_{1}$ when $\hat{\alpha}>\gamma_{t h}>0 ; \mathrm{P}_{\mathrm{e}}^{\hat{\alpha}}<\kappa_{2}<\kappa_{1}$ when $\alpha>\hat{\alpha}>\theta_{\text {th }}>\gamma_{t h}$; and $\mathrm{P}_{\mathrm{e}}^{\hat{\alpha}}=$ $G(\sqrt{A}, \alpha)$ when $\hat{\alpha}>\beta_{\text {th }}>\alpha$.

Then, stochastic signaling performs worse than conventional signaling if the standard deviation $\varepsilon$ of the channel coefficient error satisfies the following inequality:

$$
\begin{gathered}
\left(\frac{1}{2}-\kappa_{1}\right) Q\left(\frac{\alpha+\gamma_{t h}}{\varepsilon}\right)+\left(\kappa_{1}-\kappa_{2}\right)\left(Q\left(\frac{2 \alpha}{\varepsilon}\right)-Q\left(\frac{\alpha+\theta_{t h}}{\varepsilon}\right)\right) \\
+\frac{1}{2} Q\left(\frac{\alpha}{\varepsilon}\right)+Q\left(\frac{\beta_{t h}-\alpha}{\varepsilon}\right) G(\sqrt{A}, \alpha) \geqslant G(\sqrt{A}, \alpha)
\end{gathered}
$$

and stochastic signaling performs better than conventional signaling if $\varepsilon$ satisfies the following inequality ${ }^{2}$ :

$$
\begin{aligned}
& \frac{1}{2}\left(\kappa_{1}+\kappa_{2}+Q\left(\frac{\alpha}{\varepsilon}\right)\right)+\left(\frac{1}{2}-\kappa_{1}\right) Q\left(\frac{\alpha-\gamma_{t h}}{\varepsilon}\right) \\
& \quad-\kappa_{1} Q\left(\frac{\beta_{t h}-\alpha}{\varepsilon}\right)+\left(\kappa_{1}-\kappa_{2}\right) Q\left(\frac{\alpha-\theta_{t h}}{\varepsilon}\right) \\
& \quad+\left(Q\left(\frac{\beta_{t h}-\alpha}{\varepsilon}\right)-Q\left(\frac{\alpha+\beta_{t h}}{\varepsilon}\right)\right) G(\sqrt{A}, \alpha) \\
& \leqslant G(\sqrt{A}, \alpha) .
\end{aligned}
$$

Proof. Please see Appendix A.1.

Although the results in Proposition 1 are presented for channel coefficient errors with a zero-mean Gaussian distribution, they can easily be extended for any type of probability distribution as well. For example, consider a generic PDF for the channel coefficient error, which is denoted by $p_{\eta}(\cdot)$. The corresponding cumulative distribution function (CDF) $F_{\eta}(\cdot)$ can be expressed as $F_{\eta}(x)=\int_{-\infty}^{x} p_{\eta}(t) d t$. Then, the results in Proposition 1 are valid when $Q(x / \varepsilon)$ in (15) and (16) are replaced by $1-F_{\eta}(x)$.

As discussed before, $G(x, k)$ can be inferred as the probability of deciding symbol 0 instead of symbol 1 , when the value of the channel coefficient is $k$, and $S_{1}=x$. In general, for a specific channel coefficient, when a larger signal value is employed, a lower probability of error can be obtained; hence, $G(x, k)$ is usually a decreasing function of $x$ in practice. Moreover, $G(x, k)=1-G(-x, k)$ can be satisfied when the channel noise has a symmetric PDF, i.e., $p_{N}(x)=p_{N}(-x)$, and the decision regions of the detector at the receiver are symmetric $\left(\Gamma_{0}=-\Gamma_{1}\right)$. In fact, the channel noise is symmetric in most practical scenarios, and some receivers such

\footnotetext{
1 If it is negative, one can redefine function $G$ in (10) by using $p_{N}(y+k x)$ instead of $p_{N}(y-k x)$

2 Note that the choice of parameters in the conditions of Proposition 1 is important to satisfy the inequalities in (15) and (16). Also, the $Q$-function is defined as $Q(x)=\left(\int_{x}^{\infty} \mathrm{e}^{-t^{2} / 2} d t\right) / \sqrt{2 \pi}$.
}

as the sign detector or the optimal MAP detector for symmetric signaling under symmetric channel noise will have symmetric decision regions. All in all, the first condition in the proposition is expected to hold in many practical scenarios. The details of how the second condition is satisfied and how the parameters in the proposition are selected will be investigated in Section 5 .

\section{Design of stochastic signals under CSI uncertainty}

First, suppose that $p_{\alpha}(\cdot)$ denotes the PDF of the actual channel coefficient $\alpha$, where each instance of the channel coefficient resides in a certain set $\Omega$. In this section, we propose two different methods for designing the stochastic signals under CSI uncertainty in the transmitter, and evaluate the performance of each method in Section 5.

\subsection{Robust stochastic signaling}

In this part, a robust design of optimal stochastic signals is presented under CSI uncertainty at the transmitter. Suppose that $\Omega$ is given by $\Omega=\left[\alpha_{0}, \alpha_{1}\right]$, that is, the channel coefficient $\alpha$ takes values in the interval of $\left[\alpha_{0}, \alpha_{1}\right]$, where $\alpha_{0}<\alpha_{1}$. It is assumed that the transmitter has the knowledge of set $\Omega$. Note that this can be realized, for example, via feedback from the receiver to the transmitter. In robust stochastic signaling, signals are designed in such a way that they minimize the average probability of error for the worst-case channel coefficient, that is, the one which maximizes the average probability of error for the transmitted signals. For this design criterion, the optimal stochastic signaling problem in (8) can be expressed as a minimax problem as follows:

$$
\begin{aligned}
& \min _{p_{S_{0}}, p_{S_{1}}} \max _{\alpha \in\left[\alpha_{0}, \alpha_{1}\right]} \sum_{i=0}^{1} \pi_{i} \int_{-\infty}^{\infty} p_{S_{i}}(x) \int_{\Gamma_{1-i}} p_{N}(y-\alpha x) d y d x \\
& \text { subject to } \mathrm{E}\left\{\left|S_{i}\right|^{2}\right\} \leqslant A .
\end{aligned}
$$

The problem in (17) can be difficult to solve in general. In the following, it is shown that in most practical scenarios, this problem can be reduced to a simpler form and the optimal signal PDFs can be obtained by solving a simpler optimization problem:

Proposition 2. The minimax problem in (17) is equivalent to the stochastic signaling problem for channel coefficient $\alpha_{0}$, that is,

$$
\begin{aligned}
& \min _{p_{S_{0}}, p_{S_{1}}} \sum_{i=0}^{1} \pi_{i} \int_{-\infty}^{\infty} p_{S_{i}}(x) \int_{\Gamma_{1-i}} p_{N}\left(y-\alpha_{0} x\right) d y d x \\
& \text { subject to } \mathrm{E}\left\{\left|S_{i}\right|^{2}\right\} \leqslant A
\end{aligned}
$$

when the following conditions are satisfied:

- $G(x, \alpha)$ is a strictly decreasing function of $x$ for any $\alpha \in\left[\alpha_{0} \alpha_{1}\right]$.

- $G(x, \alpha)$ is a strictly decreasing (increasing) function of $\alpha$ for all $x>0(x<0)$.

Proof. Please see Appendix A.2.

Proposition 2 states that, under certain sufficient conditions, the robust design of stochastic signals becomes equivalent to the stochastic signal design for the smallest magnitude of the channel coefficient in set $\Omega$. (It is important to note that this conclusion is not true in general if the conditions in the proposition are not satisfied; that is, in some cases, a larger channel coefficient may have worse performance than a smaller channel coefficient in the presence of non-Gaussian noise.) The simplified problem in (18) 
has a well-known structure, which was investigated for example in [10]. The problem can be solved separately for $S_{0}$ and $S_{1}$ by expressing the problem as two decoupled optimization problems. Then it can be shown that if $G\left(S_{i}, \alpha_{0}\right)$ is a continuous function of $S_{i}$ and $S_{i}$ takes values in $[-\gamma, \gamma]$ for some finite positive $\gamma$, then each optimal signal PDF $p_{S_{i}}$ can be represented by a randomization between at most two signal levels as in (12) [10,31].

It is also noted that if $\left[\alpha_{0}, \alpha_{1}\right]$ is a positive interval, then the two conditions in Proposition 2 can be reduced to a single condition. Suppose that $u=\alpha x$. Then, $G(x, \alpha)$ can be written as $G(u)=\int_{\Gamma_{0}} p_{N}(y-u) d y$. Therefore, if $\alpha$ is positive, then the conditions in Proposition 2 are equivalent to that $G(u)$ is a decreasing function of $u$.

After obtaining the optimal signal PDFs $p_{S_{0}}$ and $p_{S_{1}}$ by solving (18), the conditional average probability of error for a given $\alpha \in \Omega$ can be calculated as

$\mathrm{P}_{\text {robu }}^{\alpha}=\sum_{i=0}^{1} \pi_{i} \int_{-\infty}^{\infty} p_{S_{i}}(x) \int_{\Gamma_{1-i}} p_{N}(y-\alpha x) d y d x$.

Finally, the average probability of error for robust stochastic signaling can be calculated as

$\mathrm{P}_{\text {robu }}=\int_{\Omega} p_{\alpha}(a) \mathrm{P}_{\text {robu }}^{a} d a$.

Note that while calculating the conditional average probability of error for a given $\alpha$, the same signal PDF is used for all $\alpha$ values, since the optimal signal PDFs do not depend on the value of the actual channel coefficient $\alpha$, but only depend on the lower boundary point of the set $\Omega$ in the robust stochastic signaling approach under the conditions in Proposition 2.

\subsection{Stochastic signaling with averaging}

In robust stochastic signaling, signal PDFs are designed for the worst-case channel coefficient, which belongs to a certain set $\Omega$. In this section, an alternative way of designing stochastic signals under CSI uncertainty is discussed. In this method, the transmitter assumes that the channel coefficient is distributed according to a PDF $\hat{p}_{\alpha}(\cdot){ }^{3}$ Then, optimal signal PDFs are designed in such a way that the average probability of error is minimized for this assumed CSI statistics under the average power constraints. This can be formulated as follows:

$$
\begin{aligned}
& \min _{p_{S_{0}}, p_{S_{1}}} \int_{-\infty}^{\infty} \hat{p}_{\alpha}(a) \sum_{i=0}^{1} \pi_{i} \int_{-\infty}^{\infty} p_{S_{i}}(x) \int_{\Gamma_{1-i}} p_{N}(y-a x) d y d x d a \\
& \text { subject to } \mathrm{E}\left\{\left|S_{i}\right|^{2}\right\} \leqslant A .
\end{aligned}
$$

Specifically, by using the statistical information about the CSI at the transmitter, we aim to obtain the optimal stochastic signals that minimize the expected value of the error probability over the distribution of the imperfect channel coefficient. As mentioned in Remark 1, we consider slow fading channels so that the statistical information about the CSI is constant for a number of bit durations.

It is noted that the problem in (21) is separable over $S_{0}$ and $S_{1}$ as well. Therefore, one can consider the optimal signals for symbol 0 and symbol 1 separately. Specifically, the optimal signal PDF for symbol 1 can be obtained by solving the following problem:

\footnotetext{
3 Note that this will not be the actual PDF of the channel coefficient in general due to CSI uncertainty at the transmitter.
}

$$
\begin{aligned}
& \min _{p_{S_{1}}} \int_{-\infty}^{\infty} \hat{p}_{\alpha}(a) \int_{-\infty}^{\infty} p_{S_{1}}(x) \int_{\Gamma_{0}} p_{N}(y-a x) d y d x d a \\
& \text { subject to } \mathrm{E}\left\{\left|S_{1}\right|^{2}\right\} \leqslant A .
\end{aligned}
$$

Changing the order of the first and the second integrals in (22), the following formulation can be obtained:

$$
\begin{aligned}
& \min _{p_{S_{1}}} \int_{-\infty}^{\infty} p_{S_{1}}(x) \int_{-\infty}^{\infty} \hat{p}_{\alpha}(a) G(x, a) d a d x \\
& \text { subject to } \mathrm{E}\left\{\left|S_{1}\right|^{2}\right\} \leqslant A
\end{aligned}
$$

where $G(x, a)$ is as defined in (10). In addition, if $H(x)$ is defined as $H(x) \triangleq \int_{-\infty}^{\infty} \hat{p}_{\alpha}(a) G(x, a) d a=\mathrm{E}\{G(x, a)\}$, where the expectation is over the assumed PDF of the channel coefficient, then (23) becomes

$\min _{p_{S_{1}}} \mathrm{E}\left\{H\left(S_{1}\right)\right\} \quad$ subject to $\mathrm{E}\left\{\left|S_{1}\right|^{2}\right\} \leqslant A$.

For this problem, it can be concluded that, under most practical scenarios, the optimal signal PDF can be characterized by a randomization between at most two distinct signal levels similarly to the previous results. Also, the optimal signal PDF for symbol 0 can be obtained similarly.

In the stochastic signaling with averaging approach, the transmitter assigns different weights to different values of the channel coefficient and designs signals based on this averaging operation over possible channel coefficient values. For example, instead of directly using the distorted channel coefficient $\hat{\alpha}$ in the signal design as in Section 3.1, the transmitter may assume a legitimate PDF around $\hat{\alpha}$ for the channel coefficient and design the stochastic signals. The performance of this approach and the other approaches is compared in the next section.

Remark 2. In practice, the proposed approaches can be applied to communications systems that operate in slow fading channels as follows. First, the transmitter sends a number of training bits to the receiver for synchronization and channel estimation purposes. During this phase, the receiver estimates the channel coefficient $\alpha$, and sends it to the transmitter via feedback. (If there is two-way communication via time-division multiplexing, the reciprocity of the channel can be utilized and the transmitter can obtain the channel coefficient information without feedback [28].) Next, the transmitter performs stochastic signal design according to one of the proposed approaches, and obtains the parameters of the optimal stochastic signals. Then, the stochastic signaling approach can be implemented via time sharing. For example, if symmetric signaling is used (i.e., $S_{0}=-S_{1}$ ) and the stochastic signal for bit 1 is represented by $p_{S_{1}}(s)=0.5 \delta(s-1.2)+0.5 \delta(s-0.75)$, then signal amplitude 1.2 is transmitted for half of bit 1 's and 0.75 is transmitted for the remaining half (similarly, -1.2 and -0.75 for bit 0's).

Depending on the previous knowledge and the channel estimation technique, one of the robust stochastic signaling or stochastic signaling with averaging approaches can be employed. When the channel estimation error is known to be bounded, an interval of $\left[\alpha_{0}, \alpha_{1}\right]$ can be specified as in Section 4.1. Otherwise, a distribution can be assumed for the channel coefficient error, which is commonly modeled by a Gaussian random variable (e.g., [32,33]), and the approach in this section can be used. The robust stochastic signaling approach takes a conservative approach and performs the design for the worst-case channel coefficient value under the conditions in Proposition 2. However, the stochastic signaling with averaging approach performs the design based on the available probability distribution of the channel coefficient. 
Remark 3. The following observations can be made when the design techniques in Section 3.1 and Section 4 are compared. The approach in Section 3.1 directly employs the noisy channel coefficient information at the transmitter, $\hat{\alpha}$, in the design of stochastic signals (see (8)). On the other hand, the robust stochastic signaling and stochastic signaling with averaging approaches in Section 4 perform the design based on the worst-case channel coefficient value and on an average channel coefficient distribution, respectively. These approaches assume that some additional information is available about the noisy channel estimate such as bounds on the estimation error, or its probability distribution. For cases in which the estimation error is not expected to be higher than a certain amount, the channel coefficient can be modeled to lie in an interval such as $\left[\alpha_{0}, \alpha_{1}\right]$, which can be obtained by using the channel estimate and the upper and lower bounds on the estimation error. Then, robust stochastic signaling performs a design for the worst-case channel coefficient, $\alpha_{0}$. When such upper and lower bounds are not available or when the conservative approach of performing a design for the worst-case channel coefficient is not desirable, the stochastic signaling with averaging approach can be utilized by assuming a probability distribution $\hat{p}_{\alpha}$ for the noisy channel coefficient, such as the Gaussian distribution [32,33]. The robust stochastic signaling and stochastic signaling with averaging approaches in Section 4 reduce to the approach in Section 3.1 that directly uses the noisy channel estimate in the stochastic signal design if $\alpha_{0}=\alpha_{1}=\hat{\alpha}$ for robust stochastic signaling (see Section 4.1) and $\hat{p}_{\alpha}(a)=\delta(a-\hat{\alpha})$ for stochastic signaling with averaging (see the beginning of this section), where $\hat{\alpha}$ is the noisy channel coefficient information at the transmitter. Since the channel coefficient information can include large errors in some cases, the design of stochastic signals based directly on the noisy channel coefficient can result in large errors as observed in the next section. Hence, the approaches in Section 4 are commonly more preferable.

\section{Performance evaluation}

In this section, two numerical examples are presented in order to investigate the theoretical results in the previous sections. In the first numerical example, we compare the performance of conventional signaling and stochastic signaling in the presence of channel coefficient errors and observe the effects of CSI uncertainty on stochastic signaling. In the second example, we evaluate the performance of the proposed design methods in Section 4. In both of the examples, a binary communications system with equally likely symbols are considered $\left(\pi_{0}=\pi_{1}=0.5\right)$, the average power limit in (4) is set to $A=1$, and the decision rule at the receiver is specified by $\Gamma_{0}=(-\infty, 0]$ and $\Gamma_{1}=[0, \infty)$ (i.e., the sign detector). Also the noise in (1) is modeled by a Gaussian mixture noise [6] with its PDF being given by $p_{N}(n)=(\sqrt{2 \pi} \sigma)^{-1} \times$ $\sum_{l=1}^{L} v_{l} \exp \left\{-\left(n-\mu_{l}\right)^{2} /\left(2 \sigma^{2}\right)\right\}$. Gaussian mixture noise is encountered in practical systems in the presence of interference [6]. For the channel noise and the detector structure as described above, $G(x, k)$ in (10) can be calculated as

$G(x, k)=\sum_{l=1}^{L} v_{l} Q\left(\frac{k x+\mu_{l}}{\sigma}\right)$.

In the first example, the mass points $\mu_{l}$ are located at $\boldsymbol{\mu}=$

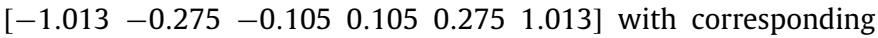
weights $\mathbf{v}=\left[\begin{array}{llllll}0.043 & 0.328 & 0.129 & 0.129 & 0.328 & 0.043\end{array}\right]$. Also each component of the Gaussian mixture noise has the same variance $\sigma^{2}$ and the average power of the noise can be calculated as $\mathrm{E}\left\{n^{2}\right\}=\sigma^{2}+0.1407$.

The channel coefficient information at the transmitter is modeled as $\hat{\alpha}=\alpha+\eta$, where $\alpha=1$ and $\eta$ is a zero-mean Gaussian

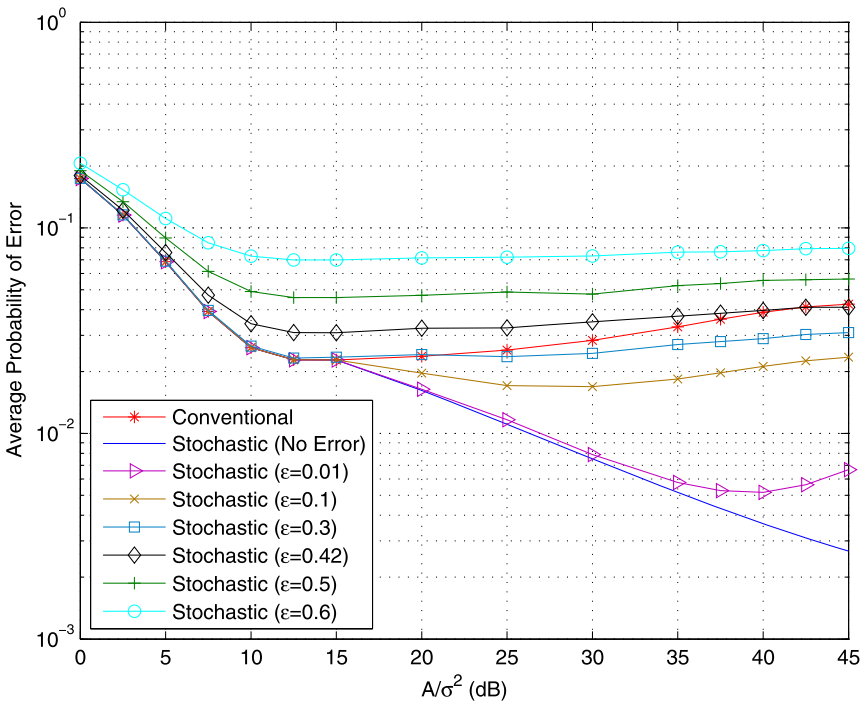

Fig. 1. Average probability of error versus $A / \sigma^{2}$ for conventional signaling and stochastic signaling with various $\varepsilon$ values.

random variable with standard deviation $\varepsilon$. Due to the symmetry of the problem, the conditional probability of error expression in (14) also provides the average probability of error in this scenario. In order to evaluate that expression, 100 realizations are obtained for $\hat{\alpha}$. Then, the optimization problem in (11) is solved for each realization and the optimal signal PDFs that are in the form of (12) are obtained by using the PSO algorithm [34]. For the details of the PSO parameters employed in this study, please refer to [12].

In Fig. 1, the average probabilities of error are plotted versus $A / \sigma^{2}$ for conventional signaling, stochastic signaling with no channel coefficient errors $(\varepsilon=0)$, and stochastic signaling with various levels of channel coefficient errors (see (11)). It is noted that the average probability of error increases as $A / \sigma^{2}$ increases after a certain value for conventional signaling and stochastic signaling with channel coefficient errors. This seemingly counterintuitive result is because of the facts that the average probabilities of error are related to the area under the shifted noise PDFs as in (5), (13) and (14), and that the noise has a multimodal PDF [12]. ${ }^{4}$ Also, it is observed that for high $A / \sigma^{2}$ values, the best performance is obtained by stochastic signaling with perfect CSI and the performance of stochastic signaling gets worse as the variance of the channel coefficient error increases. Another observation is that for low values of $\varepsilon$, stochastic signaling still performs better than conventional signaling for high $A / \sigma^{2}$ values and their performance is similar for high $\sigma^{2}$, i.e., when $A / \sigma^{2}$ is smaller than $15 \mathrm{~dB}$. In fact, one can calculate the average probability of error analytically for low $A / \sigma^{2}$ values for each $\varepsilon$, as discussed in [1]. In addition, we can apply the conditions in Proposition 1 and check if the conventional signaling is improvable or nonimprovable via stochastic signaling for given $\varepsilon$ values. Firstly, we examine the first condition in the proposition. $G(x, k)$ is as expressed in (25) for this example and it is a convex combination of $Q$ functions. Therefore, $G(x, k)$ is a strictly decreasing function of $x$ as $Q(x)$ is a monotone decreasing function. Also, since $Q(x)=1-Q(-x)$ and the components of Gaussian mixture noise are symmetric, we have $G(x, k)=1-G(-x, k)$ as well. Hence, the first condition

\footnotetext{
4 Since signals are designed according to noisy channel coefficients in stochastic signaling with channel coefficient errors, noise PDFs may not be shifted in an optimal way to minimize the area under the shifted PDFs. Therefore, that area may not be a monotonic function of $A / \sigma^{2}$, and can increase in some cases as $A / \sigma^{2}$ increases.
} 


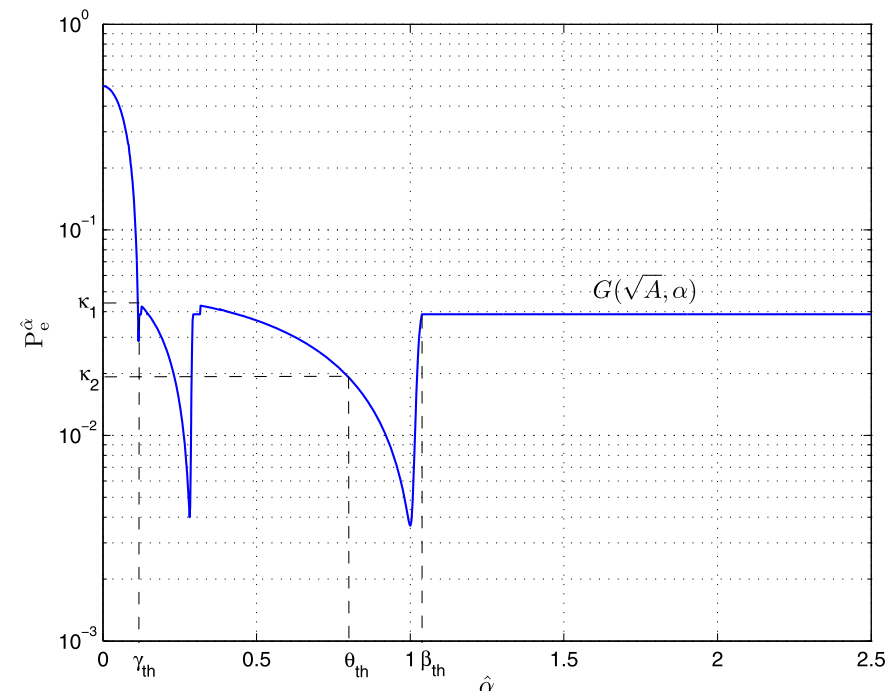

Fig. 2. $\mathrm{P}_{\mathrm{e}}^{\hat{\alpha}}$ versus $\hat{\alpha}$ for $A / \sigma^{2}=40 \mathrm{~dB}$. The second condition in Proposition 1 is satisfied for $\kappa_{1}=0.04354, \kappa_{2}=0.01913, \gamma_{\text {th }}=0.1135, \theta_{\text {th }}=0.8, \beta_{\text {th }}=1.038$, and $G(\sqrt{A}, \alpha)=0.03884$.

in Proposition 1 is satisfied. In order to check the second condition, the plot of $\mathrm{P}_{\mathrm{e}}^{\hat{\alpha}}$ versus $\hat{\alpha}$ is presented in Fig. 2 for $A / \sigma^{2}=40 \mathrm{~dB}$. It is observed that $\mathrm{P}_{\mathrm{e}}^{\hat{\alpha}}$ does not have a monotonic structure; that is, it increases, decreases or remains the same as $\hat{\alpha}$ increases. However, it obeys the structure specified in the second condition of Proposition 1. Specifically, when $\hat{\alpha}>\gamma_{t h}=0.1135, \mathrm{P}_{\mathrm{e}}^{\hat{\alpha}}$ is less than $\kappa_{1}=0.04354$, and when $\theta_{\text {th }}=0.8<\hat{\alpha}<\alpha=1$, $\mathrm{P}_{\mathrm{e}}^{\hat{\alpha}}$ becomes less than $\kappa_{2}=0.01913$, which is even smaller than $\kappa_{1}$. Also, when $\hat{\alpha}>\beta_{\text {th }}=1.038$, $\mathrm{P}_{\mathrm{e}}^{\hat{\alpha}}$ becomes equal to $G(\sqrt{A}, \alpha)=0.03884$, which is the average probability of error for conventional signaling. The values of $\kappa_{1}, \kappa_{2}, \gamma_{t h}, \theta_{t h}$, and $\beta_{t h}$ are illustrated in Fig. 2. Based on the specified parameters, (15) becomes

$$
\begin{gathered}
0.45646 Q\left(\frac{1.1135}{\varepsilon}\right)+0.02441\left(Q\left(\frac{2}{\varepsilon}\right)-Q\left(\frac{1.8}{\varepsilon}\right)\right) \\
+0.5 Q\left(\frac{1}{\varepsilon}\right)+0.03884 Q\left(\frac{0.038}{\varepsilon}\right) \geqslant 0.03884 .
\end{gathered}
$$

For $\varepsilon=0.6$, the left-hand side of this inequality is calculated to be 0.0568 ; hence, the inequality is satisfied. This means that when $A / \sigma^{2}=40 \mathrm{~dB}$, if the standard deviation of the channel coefficient error is equal to 0.6 , we can conclude that stochastic signaling is outperformed by conventional signaling. In fact, it can be observed from Fig. 1 that for $A / \sigma^{2}=40 \mathrm{~dB}$ and $\varepsilon=0.6$, the performance of stochastic signaling is quite worse than that of conventional signaling as Proposition 1 asserts. Also note that when $\varepsilon=0.5178 \triangleq \varepsilon^{*},(15)$ becomes an equality. Similarly, based on the selected parameters, it can be shown that (16) is satisfied for $\varepsilon=$ $0.3,0.1,0.01$, meaning that conventional signaling is outperformed by stochastic signaling as a result of Proposition 1 for these $\varepsilon$ values [1]. This can also be observed from Fig. 1 when $A / \sigma^{2}=40 \mathrm{~dB}$ for $\varepsilon=0.3,0.1,0.01$. Also, when $\varepsilon=0.3395 \triangleq \hat{\varepsilon}$, (16) turns out to be an equality.

In order to explore the performance of stochastic signaling in the presence of channel coefficient errors, Fig. 3 is presented. As expected, the average probability of error for stochastic signaling increases with the standard deviation of the channel coefficient error, $\varepsilon$. Therefore, in the presence of large channel coefficient errors (i.e., large $\varepsilon$ ), using conventional deterministic signaling instead of stochastic signaling can be more preferable, whereas for small channel coefficient errors, stochastic signaling can be employed to achieve smaller average probabilities of error than conventional signaling. In Fig. $3, \varepsilon^{*}$ and $\hat{\varepsilon}$ are also illustrated, together

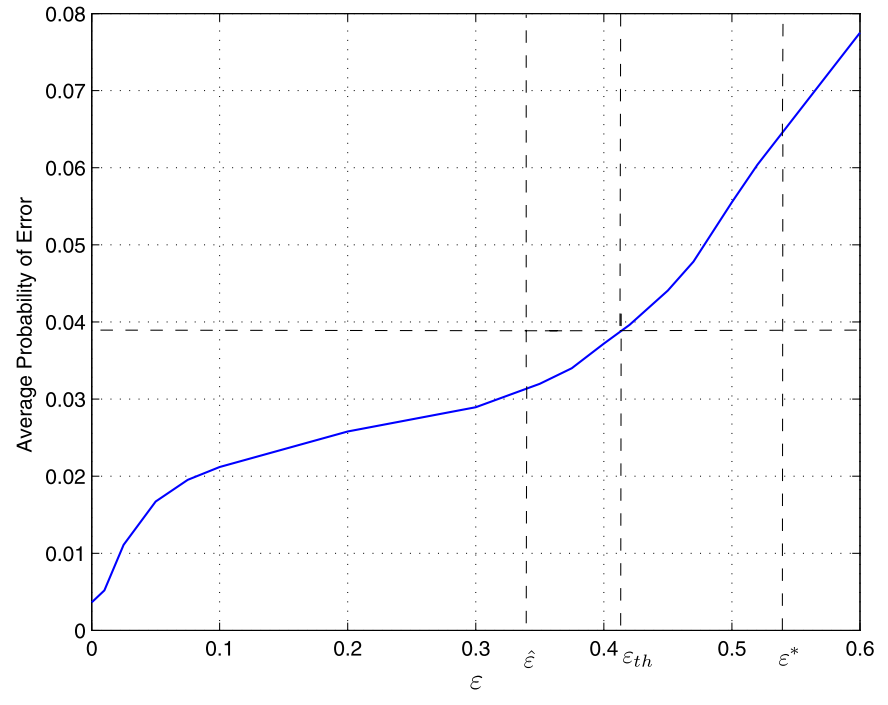

Fig. 3. Average probability of error versus $\varepsilon$ for stochastic signaling. At $\varepsilon_{t h}=0.413$, stochastic signaling has the same average probability of error as conventional signaling.

with the point $\varepsilon_{t h}$ at which the performance of stochastic signaling and conventional signaling becomes the same. It is noted that the conditions in Proposition 1 are not necessary but only sufficient conditions for the improvability and nonimprovability of conventional signal via stochastic signaling. In addition, it is observed that the performance of conventional deterministic signaling does not change with $\varepsilon$ since it always employs $S_{1}=-S_{0}=\sqrt{A}$ irrespective of the channel state information.

In the second example, the mass points $\mu_{l}$ of the Gaussian mix-

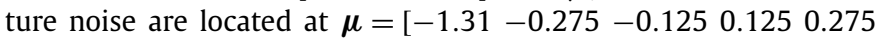
1.31] with corresponding weights $\mathbf{v}=\left[\begin{array}{llll}0.002 & 0.319 & 0.179 & 0.179\end{array}\right.$ 0.3190 .002 ]. Each component of the Gaussian mixture noise has the same variance $\sigma^{2}$ and the average power of the noise can be calculated as $\mathrm{E}\left\{n^{2}\right\}=\sigma^{2}+0.0607$. For this example, $\hat{\alpha}$ is again modeled as $\hat{\alpha}=\alpha+\eta$, where $\eta$ is a zero-mean Gaussian random variable with variance $\varepsilon^{2}$. We assume that the actual channel coefficient $\alpha$ has a uniform distribution over set $\Omega=[0.8,1.2]$; i.e., $\alpha$ is distributed as $\mathcal{U}[0.8,1.2]$.

First, we compare the average probability of error performance of different signaling strategies:

Stochastic-perfect: It is assumed that the transmitter has the knowledge of the actual channel coefficient, which is used in the signal design. In the simulations, 100 realizations are generated for a uniformly distributed $\alpha$. The optimal signal PDFs and the corresponding probabilities of error are calculated for each realization. Then, by averaging over the PDF of $\alpha$, the average probabilities of error are obtained.

Conventional: The transmitter selects the signals as $S_{1}=$ $-S_{0}=\sqrt{A}=1$. For each realization of $\alpha$, the corresponding probabilities of error are calculated and then their average is taken over the PDF of $\alpha$.

Stochastic-distorted: The transmitter has imperfect CSI and it uses a distorted (imperfect) channel coefficient $\hat{\alpha}$ directly in the design of signals, as discussed in Section 3.1. In Fig. 4, average probabilities of error are plotted for $\varepsilon=0.05$ and $\varepsilon=0.1$.

Stochastic-average: The transmitter assumes that the PDF of the channel coefficient $\hat{p}_{\alpha}(a)$ is specified by $\mathcal{N}\left(\hat{\alpha}, \Delta^{2}\right)$. Then, by solving (24), the optimal signal PDF $p_{S_{1}}^{\hat{\alpha}}$ for signal 1 can be obtained for each $\hat{\alpha}$. Next, the conditional probability of error for symbol 1 can be expressed as $\mathrm{P}_{\text {aver }}=\int_{-\infty}^{\infty} p_{\alpha}(a) \int_{-\infty}^{\infty} p_{\hat{\alpha} \mid \alpha}(\hat{a}) \times$ $\int_{-\infty}^{\infty} p_{S_{1}}^{\hat{a}}(x) G(x, a) d x d a ̂ d a$, where $p_{\hat{\alpha} \mid \alpha}(\cdot)$ is the conditional PDF of $\hat{\alpha}$ for a given $\alpha$. Note that, due to the symmetry, the conditional 


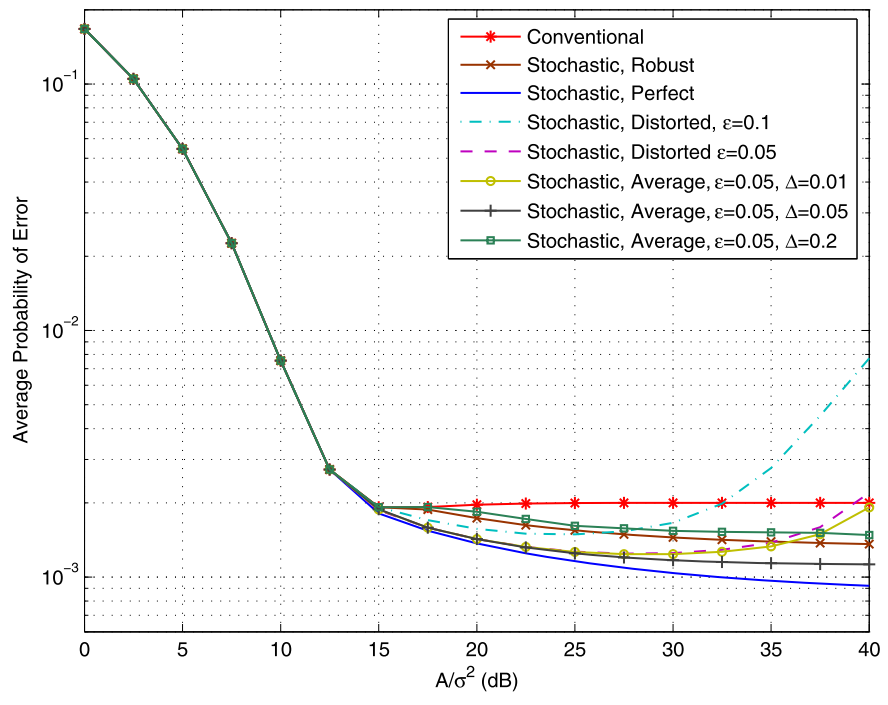

Fig. 4. Average probability of error versus $A / \sigma^{2}$ for various signaling strategies.

probability of error is equal to the average probability of error in this example as well. In Fig. 4, the average probabilities of error are plotted for $\Delta=0.01, \Delta=0.05$, and $\Delta=0.2$, where $\varepsilon=0.05$ in each case.

Stochastic-robust: First, one can show that the conditions in Proposition 2 are satisfied for this example. $G(x, \alpha)$ in (25) is a convex combination of $Q$ functions, i.e., $Q\left(\frac{\alpha x+\mu_{l}}{\sigma}\right)$. Also, since $\alpha$ is always positive $(\alpha \in[0.8,1.2]), Q\left(\frac{\alpha x+\mu_{l}}{\sigma}\right)$ is a decreasing function of $x$. In addition, it is a decreasing function of $\alpha$ if $x$ is positive, and it increases with $\alpha$ when $x$ is negative. In fact, since $[0.8,1.2]$ is a positive interval, we can write $u=\alpha x$ and $G(u)$ becomes a decreasing function of $u$ as $Q\left(\frac{u+\mu_{l}}{\sigma}\right)$ decreases with $u$. Therefore, we can apply the result in Proposition 2 in this example. That is, the optimal signal PDFs are obtained by solving (17) with $\alpha_{0}=0.8$ since $\Omega=[0.8,1.2]$. Then, the average probabilities of error are calculated via (19) and (20).

In Fig. 4, the average probabilities of error are plotted versus $A / \sigma^{2}$ for conventional signaling, stochastic signaling with perfect CSI, stochastic signaling with distorted channel coefficients, stochastic signaling with averaging, and robust stochastic signaling. It is observed that for high $\sigma^{2}$, specifically when $A / \sigma^{2}$ is smaller than $15 \mathrm{~dB}$, all signaling strategies perform similarly, and for high $A / \sigma^{2}$ values, stochastic signaling with perfect CSI achieves the best performance. The second best performance is obtained by the stochastic signaling with averaging method when the parameters are $\varepsilon=\Delta=0.05$. Although conventional signaling gives the worst performance for medium $A / \sigma^{2}$ values, the worst performance is observed for stochastic signaling with distorted channel coefficients for high $A / \sigma^{2}$ values. Robust stochastic signaling performs somewhere between stochastic signaling with perfect CSI and conventional signaling. Robust signaling performs better (worse) than stochastic signaling with averaging for $\Delta=0.2(\Delta=0.05)$ at high or medium $A / \sigma^{2}$ values. When $\varepsilon=0.05$, stochastic signaling with averaging for $\Delta=0.01$ and stochastic signaling with distorted channel coefficients perform very similarly and they achieve better performance than robust signaling for medium $A / \sigma^{2}$ values; however, their performance is worse than robust signaling for high $A / \sigma^{2}$ values.

In order to investigate the effects of $\Delta$ on the average probability of error performance of the stochastic signaling with averaging method, Fig. 5 is presented. It can be observed that setting $\Delta$ to 0.05 provides the best performance. This means that the average probability of error performance is smaller when the standard deviation of the assumed PDF of the channel coefficient $\Delta$ gets closer

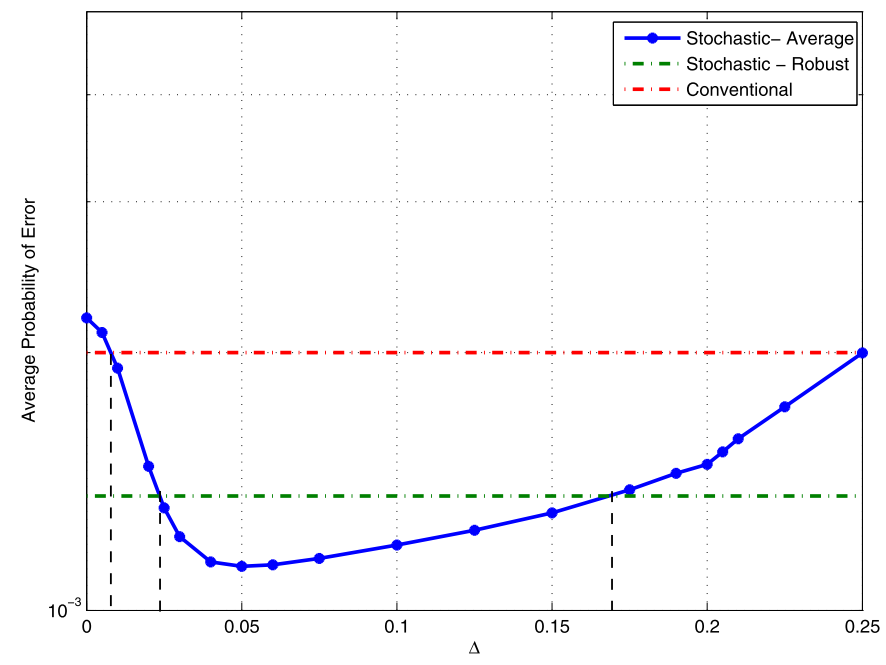

Fig. 5. Average probability of error versus $\Delta$ for stochastic signaling with averaging when $A / \sigma^{2}=40 \mathrm{~dB}$ and $\varepsilon=0.05$. Stochastic signaling with averaging performs the same as conventional signaling when $\Delta=0.0078$. It has the same average probability of error as robust stochastic signaling at $\Delta=0.0236$ and $\Delta=0.1684$.

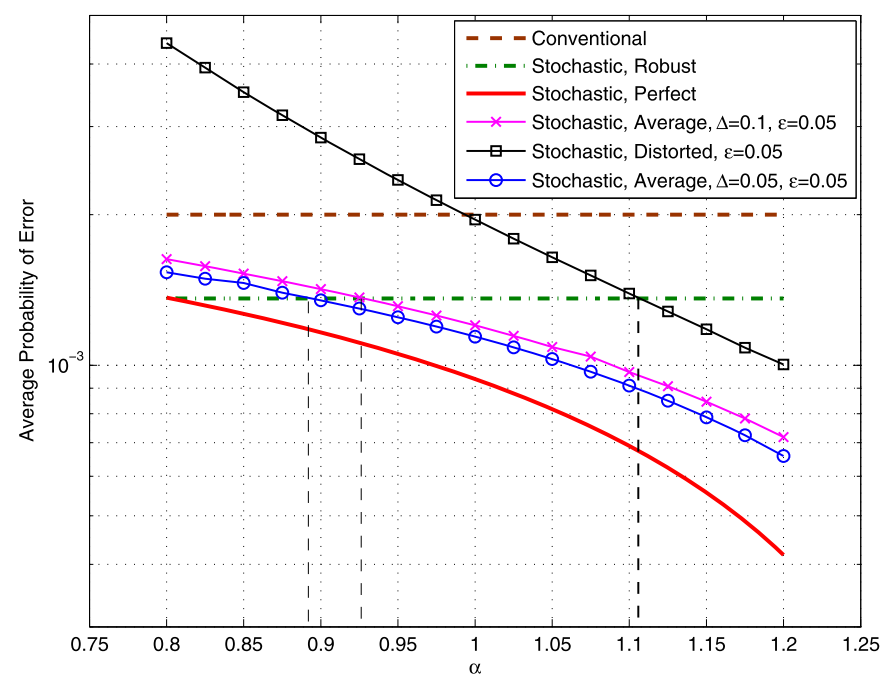

Fig. 6. Average probability of error versus $\alpha$ for various signaling strategies when $A / \sigma^{2}=40 \mathrm{~dB}$

to the standard deviation of the channel coefficient error $\varepsilon$. As we increase or decrease the value of $\Delta$ from 0.05 , the average probability of error increases. Therefore, choosing very small or very large $\Delta$ values degrades the performance of the stochastic signaling with averaging strategy. Note that $\Delta=0$ corresponds to the stochastic signaling with distorted channel coefficients method. It can be observed from Fig. 5 that if $\Delta$ is less than 0.0078 , conventional signaling which has an average probability of error of 0.002 is better than this averaging strategy. Also, if $\Delta$ is less than 0.0236 or larger than 0.1684 , robust stochastic signaling which has an average probability of error of 0.00136 achieves better performance than stochastic signaling with averaging, whereas the performance of stochastic signaling with averaging is better than robust signaling if $0.0236<\Delta<0.1684$. Therefore, it is concluded that if the variance of the channel coefficient error is estimated reasonably well, the stochastic signaling with averaging approach outperforms the other approaches.

Furthermore, we investigate in Fig. 6 the average probability of error performance of conventional signaling, stochastic signaling with perfect CSI, robust stochastic signaling, stochastic signaling with averaging when $\varepsilon=0.05$ and $\Delta=0.1$ and when 
Table 1

Optimal signal PDFs [in the form of $p_{S_{1}}(s)=\lambda_{1} \delta\left(s-s_{11}\right)+\left(1-\lambda_{1}\right) \delta\left(s-s_{12}\right)$ ] for symbol 1 according to stochastic signaling and robust stochastic signaling for various $\alpha$.

\begin{tabular}{lllll}
\hline$A / \sigma^{2}(\mathrm{~dB})$ & $\alpha$ & \multicolumn{2}{l}{ Stochastic } \\
\cline { 3 - 5 } & & $\lambda_{1}$ & $s_{11}$ & $s_{12}$ \\
\hline 10 & 0.9 & $\mathrm{~N} / \mathrm{A}$ & 1 & 1 \\
10 & 1.1 & $\mathrm{~N} / \mathrm{A}$ & 1 & 1 \\
25 & 0.9 & 0.3254 & 1.5642 & 0.5496 \\
25 & 1.1 & 0.5557 & 1.2798 & 0.4497 \\
40 & 0.9 & 0.4211 & 1.4838 & 0.3546 \\
40 & 1.1 & 0.6590 & 1.214 & 0.2901 \\
\hline$A / \sigma^{2}(\mathrm{~dB})$ & $\alpha$ & Robust & & \\
\cline { 3 - 5 } & & $\lambda_{1}$ & $s_{11}$ & $s_{12}$ \\
\hline 10 & $\mathrm{~N} / \mathrm{A}$ & $\mathrm{N} / \mathrm{A}$ & 1 & 1 \\
25 & $\mathrm{~N} / \mathrm{A}$ & 0.2276 & 1.7597 & 0.6183 \\
40 & $\mathrm{~N} / \mathrm{A}$ & 0.3200 & 1.6693 & 0.3989 \\
\hline
\end{tabular}

$\varepsilon=\Delta=0.05$, and stochastic signaling with distorted channel coefficients when $\varepsilon=0.05$ versus the actual value of the channel coefficient $\alpha$ at $A / \sigma^{2}=40 \mathrm{~dB}$. We observe that the average probability of error decreases as $\alpha$ increases for all strategies. ${ }^{5}$ For each value of the channel coefficient, the lower bound for the probability of error is obtained by stochastic signaling with perfect CSI. For small values of $\alpha$, i.e., when $\alpha<0.894$, robust stochastic signaling is better than stochastic signaling with averaging even for $\Delta=\varepsilon$. However, for larger $\alpha$ values, such as for $\alpha>1.107$, robust signaling performs worse than stochastic signaling with averaging and stochastic signaling with distorted channel coefficients. This shows that since the signals are designed for $\alpha_{0}=0.8$ in robust stochastic signaling, when the actual $\alpha$ is close to 0.8 , robust signaling achieves improved performance. Performance of stochastic signaling with averaging is better than conventional signaling and stochastic signaling with distorted channel coefficients for every $\alpha$ value. Although conventional signaling yields larger average probabilities of error than stochastic signaling with distorted channel coefficients for $\alpha>0.9935$, employing distorted channel coefficients in the signal design results in the worst average probability of error performance when $\alpha$ has a smaller value.

Finally, in order to provide additional explanations of the preceding results, Table 1 and Table 2 are presented. In Table 1, the optimal signals for robust stochastic signaling and stochastic signaling for the given channel coefficient value $\alpha$ are presented for various $A / \sigma^{2}$ values. Note that in robust signaling the actual value of $\alpha$ is irrelevant since all the signals are designed for $\alpha=0.8$. It is observed that when $A / \sigma^{2}=10 \mathrm{~dB}$, both strategies have the same solution as the conventional signaling. However, as $A / \sigma^{2}$ increases, the randomization between two signal values becomes more effective and this may help reduce the average probability of error. For example, when $A / \sigma^{2}=25 \mathrm{~dB}$, the average probability of error for robust signaling is 0.00155 , whereas it is 0.00199 for conventional signaling. In Table 2 , the optimal signals for stochastic signaling with averaging when $A / \sigma^{2}=40 \mathrm{~dB}$ are presented. Note that the assumed PDF of the channel coefficient in that strategy is $\mathcal{N}\left(\hat{\alpha}, \Delta^{2}\right)$. It is observed that when $\Delta$ is very small, i.e., $\Delta=0.01$, the optimal signal PDFs are close to the optimal signal PDFs of the stochastic signaling case given in Table 1 . Also, when $\hat{\alpha}=0.9$ and $\Delta=0.2$, the optimal signal PDF is close to that for conventional signaling since the optimal PDF has a mass point at 0.9684 with a weight of 0.9302 .

\footnotetext{
5 Although it is not very clear in Fig. 6, the average probabilities of error for conventional signaling and robust signaling also slightly decrease as $\alpha$ increases. The reason for the almost constant performance is that the designed signals for these approaches around $A / \sigma^{2}=40 \mathrm{~dB}$ cannot mitigate the effect of the largest component of the Gaussian mixture noise, which is located at 1.31 .
}

Table 2

Optimal signal PDFs [in the form of $p_{S_{1}}(s)=\lambda_{1} \delta\left(s-s_{11}\right)+\left(1-\lambda_{1}\right) \delta\left(s-s_{12}\right)$ ] for symbol 1 according to stochastic signaling with averaging when $A / \sigma^{2}=40 \mathrm{~dB}$.

\begin{tabular}{lllll}
\hline$\hat{\alpha}$ & $\Delta$ & \multicolumn{3}{l}{ Averaging } \\
\cline { 3 - 5 } & & $\lambda_{1}$ & $s_{11}$ & $s_{12}$ \\
\hline 0.9 & 0.01 & 0.41 & 1.5016 & 0.3575 \\
0.9 & 0.05 & 0.351 & 1.5922 & 0.4114 \\
0.9 & 0.2 & 0.0698 & 1.3519 & 0.9684 \\
1.1 & 0.01 & 0.6466 & 1.2247 & 0.2917 \\
1.1 & 0.05 & 0.575 & 1.2892 & 0.323 \\
1.1 & 0.2 & 0.476 & 1.2815 & 0.6453 \\
\hline
\end{tabular}

\section{Concluding remarks}

In this study, the effects of imperfect CSI on stochastic signaling and the design of stochastic signals in the presence of CSI uncertainty have been investigated. Regarding the comparison between the proposed stochastic signaling approaches, robust stochastic signaling requires less amount of statistical information about the channel coefficient error than stochastic signaling with averaging since the former uses only the smallest channel coefficient value in the signal design while an estimate for the PDF of the channel coefficient error is needed in the latter. However, the use of the smallest channel coefficient value in robust stochastic signaling can result in poor performance when the probability of having very small channel coefficients is nonzero. Therefore, in practice, it can be useful to consider only the channel coefficient values with significant probabilities in determining the smallest channel coefficient. In addition, the numerical examples have indicated that the stochastic signaling with averaging approach performs better than the other practical approaches as long as the statistics of the channel coefficient error are estimated reasonably well. However, its computational complexity is higher than that of robust stochastic signaling as an averaging operation is performed over the channel coefficient.

\section{Appendix A}

\section{A.1. Proof of Proposition 1}

In the following, lower and upper bounds for the expression in (14) are derived in order to prove the statements in the proposition. We start by noticing the fact that the sign of the channel coefficient knowledge at the transmitter is important. Suppose that $p_{S_{1}}^{\hat{\alpha}}$ is the optimal PDF obtained from (11) for a given $\hat{\alpha}$. Therefore, if $-\hat{\alpha}$ is used instead of $\hat{\alpha}$, then $p_{S_{1}}^{-\hat{\alpha}}$ will be the optimal solution of (11) and the value of $p_{S_{1}}^{-\hat{\alpha}}(x)$ will be equal to $p_{S_{1}}^{\hat{\alpha}}(-x)$. This observation can be utilized in (13), and also using the fact that $G(x, k)=1-G(-x, k), \mathrm{P}_{\mathrm{e}}^{\hat{\alpha}}=1-\mathrm{P}_{\mathrm{e}}^{-\hat{\alpha}}$ can be obtained as follows:

$$
\begin{aligned}
\int_{-\infty}^{\infty} p_{S_{1}}^{\hat{\alpha}}(x) G(x, k) d x & =\int_{-\infty}^{\infty} p_{S_{1}}^{-\hat{\alpha}}(-x)(1-G(-x, k)) d x \\
& =\int_{-\infty}^{\infty} p_{S_{1}}^{-\hat{\alpha}}(t)(1-G(t, k)) d t \\
& =1-\int_{-\infty}^{\infty} p_{S_{1}}^{-\hat{\alpha}}(t) G(t, k) d t=1-\mathrm{P}_{\mathrm{e}}^{-\hat{\alpha}}
\end{aligned}
$$

It is stated in the second condition of the proposition that $\mathrm{P}_{\mathrm{e}}^{\hat{\alpha}}<\kappa_{1}$ when $\hat{\alpha}>\gamma_{t h}$, and $\mathrm{P}_{\mathrm{e}}^{\hat{\alpha}}<\kappa_{2}<\kappa_{1}$ when $\alpha>\hat{\alpha}>\theta_{t h}$. Therefore, if we insert $-\hat{\alpha}$ instead of $\hat{\alpha}$ in these conditions, we get $\mathrm{P}_{\mathrm{e}}^{-\hat{\alpha}}<\kappa_{1}$ when $-\hat{\alpha}>\gamma_{\text {th }}$ and $\mathrm{P}_{\mathrm{e}}^{-\hat{\alpha}}<\kappa_{2}<\kappa_{1}$ when $\alpha>-\hat{\alpha}>\theta_{\text {th }}$. Using 
the result in (A.1) and rearranging the terms yield $\mathrm{P}_{\mathrm{e}}^{\hat{\alpha}}>1-\kappa_{1}$ when $\hat{\alpha}<-\gamma_{\text {th }}$ and $\mathrm{P}_{\mathrm{e}}^{\hat{\alpha}}>1-\kappa_{2}>1-\kappa_{1}$ when $-\alpha<\hat{\alpha}<-\theta_{\text {th }}$. Also, since $G(x, k)$ is a strictly decreasing function of $x$ when $k$ is positive, then $G(x, \hat{\alpha})$ is a strictly increasing function of $x$ if $\hat{\alpha}$ is negative. Therefore, for a given $\hat{\alpha}<0$, the optimal signal PDF $p_{S_{1}}^{\hat{\alpha}}$ assigns the weights on negative numbers instead of positive ones since for each positive value of $S_{1}$, its negative can be used instead, which results in the same average power value and a smaller $\mathrm{E}\left\{G\left(S_{1}, \hat{\alpha}\right)\right\}$. Furthermore, since $G(x, \alpha)$ is a strictly decreasing function, and $G(x, \alpha)=1-G(-x, \alpha)$, we have $G(x, \alpha)>$ $G(0, \alpha)=0.5$ for $x<0$. Thus, by using these two facts and the expression in (13), we conclude that if $\hat{\alpha}<0$, then $\mathrm{P}_{\mathrm{e}}^{\hat{\alpha}}>0.5$ [and $\mathrm{P}_{\mathrm{e}}^{\hat{\alpha}}<0.5$, if $\hat{\alpha}>0$ ]. Now, one can find a lower bound on $\mathrm{P}_{\mathrm{e}}$ in (14) as follows:

$$
\begin{aligned}
& \mathrm{P}_{\mathrm{e}}=\int_{-\infty}^{\infty} p_{\hat{\alpha}}(a) \mathrm{P}_{\mathrm{e}}^{a} d a \\
& \geqslant \int_{-\infty}^{-\gamma_{t h}} p_{\hat{\alpha}}(a) \mathrm{P}_{\mathrm{e}}^{a} d a+\int_{-\gamma_{t h}}^{0} p_{\hat{\alpha}}(a) \mathrm{P}_{\mathrm{e}}^{a} d a+\int_{\beta_{t h}}^{\infty} p_{\hat{\alpha}}(a) \mathrm{P}_{\mathrm{e}}^{a} d a \\
& >\left(1-\kappa_{1}\right) \mathrm{P}\left(\hat{\alpha}<-\gamma_{t h}\right)+\left(\kappa_{1}-\kappa_{2}\right) \mathrm{P}\left(-\alpha<\hat{\alpha}<-\theta_{t h}\right) \\
& +\frac{1}{2} \mathrm{P}\left(-\gamma_{t h}<\hat{\alpha}<0\right)+\mathrm{P}\left(\beta_{t h}<\hat{\alpha}\right) G(\sqrt{A}, \alpha) \\
& =\left(1-\kappa_{1}\right) \mathrm{P}\left(\frac{\eta}{\varepsilon}>\frac{\alpha+\gamma_{t h}}{\varepsilon}\right) \\
& +\left(\kappa_{1}-\kappa_{2}\right) \mathrm{P}\left(\frac{-2 \alpha}{\varepsilon}<\frac{\eta}{\varepsilon}<\frac{-\alpha-\theta_{t h}}{\varepsilon}\right) \\
& +\frac{1}{2} \mathrm{P}\left(\frac{-\alpha}{\varepsilon}<\frac{\eta}{\varepsilon}<\frac{-\alpha-\gamma_{t h}}{\varepsilon}\right) \\
& +\mathrm{P}\left(\frac{\eta}{\varepsilon}>\frac{\beta_{t h}-\alpha}{\varepsilon}\right) G(\sqrt{A}, \alpha) \\
& =\left(1-\kappa_{1}\right) Q\left(\frac{\alpha+\gamma_{t h}}{\varepsilon}\right) \\
& +\left(\kappa_{1}-\kappa_{2}\right)\left(Q\left(\frac{2 \alpha}{\varepsilon}\right)-Q\left(\frac{\alpha+\theta_{t h}}{\varepsilon}\right)\right) \\
& +\frac{1}{2}\left(Q\left(\frac{\alpha}{\varepsilon}\right)-Q\left(\frac{\alpha+\gamma_{t h}}{\varepsilon}\right)\right)+Q\left(\frac{\beta_{t h}-\alpha}{\varepsilon}\right) G(\sqrt{A}, \alpha) \\
& =\left(\frac{1}{2}-\kappa_{1}\right) Q\left(\frac{\alpha+\gamma_{t h}}{\varepsilon}\right) \\
& +\left(\kappa_{1}-\kappa_{2}\right)\left(Q\left(\frac{2 \alpha}{\varepsilon}\right)-Q\left(\frac{\alpha+\theta_{t h}}{\varepsilon}\right)\right) \\
& +\frac{1}{2} Q\left(\frac{\alpha}{\varepsilon}\right)+Q\left(\frac{\beta_{t h}-\alpha}{\varepsilon}\right) G(\sqrt{A}, \alpha) \text {. }
\end{aligned}
$$

Note that the first inequality follows from the fact that a positive term, namely, $\int_{0}^{\beta_{t h}} p_{\hat{\alpha}}(a) \mathrm{P}_{\mathrm{e}}^{a} d a$, is removed from the initial expression $\int_{-\infty}^{\infty} p_{\hat{\alpha}}(a) \mathrm{P}_{\mathrm{e}}^{a} d a$. Also, in obtaining the first and the second terms after the second inequality, we use the fact that although $\mathrm{P}_{\mathrm{e}}^{\hat{\alpha}}>1-\kappa_{1}$ when $\hat{\alpha}<-\gamma_{t h}$, the bound is tighter, that is, $\mathrm{P}_{\mathrm{e}}^{\hat{\alpha}}>1-\kappa_{2}$, when $-\alpha<\hat{\alpha}<-\theta_{t h}<-\gamma_{t h}$. For a given $\varepsilon$, if the final expression in (A.2) is greater than or equal to $G(\sqrt{A}, \alpha)$, then $\mathrm{P}_{\mathrm{e}}>G(\sqrt{A}, \alpha)$. Therefore, under the conditions in the proposition, if the inequality in (15) is satisfied for a given value of the standard deviation $\varepsilon$ of the channel coefficient error, it is sufficient to conclude that conventional signaling performs better than stochastic signaling.
Next, the following upper bound on $\mathrm{P}_{\mathrm{e}}$ in (14) can be obtained based on a similar approach to that in obtaining (A.2) (please see [1] for details):

$$
\begin{aligned}
\mathrm{P}_{\mathrm{e}} \leqslant & \frac{1}{2}\left(\kappa_{1}+\kappa_{2}+Q\left(\frac{\alpha}{\varepsilon}\right)\right)+\left(\frac{1}{2}-\kappa_{1}\right) Q\left(\frac{\alpha-\gamma_{t h}}{\varepsilon}\right) \\
& -\kappa_{1} Q\left(\frac{\beta_{t h}-\alpha}{\varepsilon}\right)+\left(\kappa_{1}-\kappa_{2}\right) Q\left(\frac{\alpha-\theta_{t h}}{\varepsilon}\right) \\
& +\left(Q\left(\frac{\beta_{t h}-\alpha}{\varepsilon}\right)-Q\left(\frac{\alpha+\beta_{t h}}{\varepsilon}\right)\right) G(\sqrt{A}, \alpha) .
\end{aligned}
$$

For a given $\varepsilon$, if the expression in (A.3) is less than or equal to $G(\sqrt{A}, \alpha)$, then $\mathrm{P}_{\mathrm{e}}<G(\sqrt{A}, \alpha)$ is obtained. Therefore, under the conditions in the proposition, if the inequality in (16) is satisfied for a given $\varepsilon$, it is sufficient to conclude that stochastic signaling performs better than conventional signaling.

\section{A.2. Proof of Proposition 2}

The minimax problem in (17) can be expressed as follows:

$$
\begin{aligned}
& \min _{p_{S_{0}}, p_{S_{1}}} \max _{\alpha \in\left[\alpha_{0}, \alpha_{1}\right]} \pi_{1} \int_{-\infty}^{\infty} p_{S_{1}}(x) G(x, \alpha) d x \\
& +\pi_{0} \int_{-\infty}^{\infty} p_{S_{0}}(x)(1-G(x, \alpha)) d x \text { subject to } \mathrm{E}\left\{\left|S_{i}\right|^{2}\right\} \leqslant A .
\end{aligned}
$$

Assume that $S_{1}$ is a nonnegative and $S_{0}$ is a nonpositive random variable. First, it is shown that this assumption does not reduce the generality of the proof. Suppose that $p_{S_{1}}^{*}$ is the PDF of $S_{1}$ which is a nonnegative random variable, and $p_{S_{0}}^{*}$ is the PDF of $S_{0}$ which is any random variable (that is, its instances can take both positive or negative values). In the minimax problem, for given $p_{S_{0}}^{*}$ and $p_{S_{1}}^{*}$, we maximize $\pi_{1} \int_{-\infty}^{\infty} p_{S_{1}}^{*}(x) G(x, \alpha) d x+$ $\pi_{0} \int_{-\infty}^{\infty} p_{S_{0}}^{*}(x)(1-G(x, \alpha)) d x$ over $\alpha \in\left[\alpha_{0}, \alpha_{1}\right]$. Now assume that $p_{S_{1}}^{\dagger}$ is symmetric with $p_{S_{1}}^{*}$, that is, $p_{S_{1}}^{\dagger}$ is a PDF for a nonpositive random variable such that $p_{S_{1}}^{*}(-x)=p_{S_{1}}^{\dagger}(x)$. Similarly, for a given $p_{S_{0}}^{*}$ and $p_{S_{1}}^{\dagger}$, we maximize $\pi_{1} \int_{-\infty}^{\infty} p_{S_{1}}^{\dagger}(x) G(x, \alpha) d x+$ $\pi_{0} \int_{-\infty}^{\infty} p_{S_{0}}^{*}(x)(1-G(x, \alpha)) d x$ over $\alpha \in\left[\alpha_{0}, \alpha_{1}\right]$. Because of the first condition in the proposition, for every $\alpha \in\left[\alpha_{0}, \alpha_{1}\right], \int_{-\infty}^{\infty} p_{S_{1}}^{*}(x) \times$ $G(x, \alpha) d x \leqslant \int_{-\infty}^{\infty} p_{S_{1}}^{\dagger}(x) G(x, \alpha) d x$, since $G(x, \alpha)$ is a strictly decreasing function of $x$; hence, the value of the maximum for $p_{S_{1}}^{*}$ will be less than or equal to that for $p_{S_{1}}^{\dagger}$, and both PDFs will yield the same average power value because of the symmetry. Since it is a minimax problem, we look for the optimal signal PDFs $p_{S_{0}}$ and $p_{S_{1}}$ that minimize the value of the maximum. Thus, by using a nonnegative $S_{1}$, we achieve a lower maximum value as compared to a nonpositive $S_{1}$. Similarly, a nonpositive $S_{0}$ will yield a smaller maximum value as compared to a nonnegative $S_{0}$. Therefore, instead of considering all PDFs, one can just consider the PDFs of a nonpositive $S_{0}$ and a nonnegative $S_{1}$ without loss of generality under the first condition in the proposition.

Based on this fact, for any given $p_{S_{0}}$ and $p_{S_{1}}$, which are the PDFs of a nonpositive $S_{0}$ and a nonnegative $S_{1}$, respectively, we maximize $V(\alpha)=\pi_{1} \int_{0}^{\infty} p_{S_{1}}(x) G(x, \alpha) d x+\pi_{0} \int_{-\infty}^{0} p_{S_{0}}(x) \times$ $(1-G(x, \alpha)) d x$ over $\alpha \in\left[\alpha_{0}, \alpha_{1}\right]$. Define $V_{1}(\alpha)=\int_{0}^{\infty} p_{S_{1}}(x) \times$ $G(x, \alpha) d x$ and $V_{0}(\alpha)=\int_{-\infty}^{0} p_{S_{0}}(x) G(x, \alpha) d x$. Then, we maximize $V(\alpha)=\pi_{1} V_{1}(\alpha)-\pi_{0} V_{0}(\alpha)+\pi_{0}$ over $\alpha \in\left[\alpha_{0}, \alpha_{1}\right]$. Under the second condition in the proposition, $G(x, \alpha)$ is a strictly decreasing function of $\alpha, \forall x>0$, and a strictly increasing function of $\alpha$, 
$\forall x<0 .{ }^{6}$ First, assume that $p_{S_{i}}(x) \neq \delta(x)$ for $i=0,1$. Then, for every $\alpha_{i}>\alpha_{j}, G\left(x, \alpha_{i}\right)<G\left(x, \alpha_{j}\right)$ if $x>0$, and $G\left(x, \alpha_{i}\right)>G\left(x, \alpha_{j}\right)$ if $x<0$. Since $p_{S_{i}}(x)$ is always nonnegative, $\int_{0}^{\infty} p_{S_{1}}(x) G\left(x, \alpha_{i}\right) d x<$ $\int_{0}^{\infty} p_{S_{1}}(x) G\left(x, \alpha_{j}\right) d x$; that is, $V_{1}\left(\alpha_{i}\right)<V_{1}\left(\alpha_{j}\right)$. Hence, $V_{1}$ is a strictly decreasing function of $\alpha$. Similarly, $\int_{-\infty}^{0} p_{S_{0}}(x) G\left(x, \alpha_{i}\right) d x>$ $\int_{-\infty}^{0} p_{S_{0}}(x) G\left(x, \alpha_{j}\right) d x$; that is, $V_{0}\left(\alpha_{i}\right)>V_{0}\left(\alpha_{j}\right)$. So, $V_{1}$ is a strictly increasing function of $\alpha$. Then, it is concluded that $V(\alpha)$ is a strictly decreasing function of $\alpha$. Hence, for $p_{S_{0}}$ and $p_{S_{1}}$, under the conditions in the proposition, $\max _{\alpha \in\left[\alpha_{0} \alpha_{1}\right]} V(\alpha)=V\left(\alpha_{0}\right)$, meaning that the minimax problem can be reduced to the form in (18). Note that, when $p_{S_{i}}(x)=\delta(x)$, then $d V_{i}(\alpha) / d \alpha=0$. If $p_{S_{1}}(x)=p_{S_{0}}(x)=\delta(x)$, then $V(\alpha)$ becomes a constant function. Also, if one of $p_{S_{1}}(x)$ or $p_{S_{0}}(x)$ is not equal to $\delta(x), V(\alpha)$ is still a strictly decreasing function of $\alpha$. Hence $\left.\max _{\alpha \in\left[\alpha_{0}\right.} \alpha_{1}\right] V(\alpha)=$ $V\left(\alpha_{0}\right)$ holds for all possible $p_{S_{0}}$ and $p_{S_{1}}$.

\section{References}

[1] C. Goken, S. Gezici, O. Arikan, Effects of channel state information uncertainty on the performance of stochastic signaling, in: Proc. IEEE Global Telecommun. Conf., Houston, TX, 2011.

[2] H.V. Poor, An Introduction to Signal Detection and Estimation, Springer-Verlag, New York, 1994.

[3] I. Korn, J.P. Fonseka, S. Xing, Optimal binary communication with nonequal probabilities, IEEE Trans. Commun. 51 (9) (2003) 1435-1438.

[4] M. Azizoglu, Convexity properties in binary detection problems, IEEE Trans. Inf. Theory 42 (4) (1996) 1316-1321.

[5] S. Loyka, V. Kostina, F. Gagnon, Error rates of the maximum-likelihood detector for arbitrary constellations: Convex/concave behavior and applications, IEEE Trans. Inf. Theory 56 (4) (2010) 1948-1960.

[6] V. Bhatia, B. Mulgrew, Non-parametric likelihood based channel estimator for Gaussian mixture noise, Signal Process. 87 (2007) 2569-2586.

[7] S. Verdu, Multiuser Detection, 1st ed., Cambridge University Press, Cambridge, UK, 1998.

[8] M.A. Klimesh, W.E. Stark, Worst-case power-constrained noise for binary-input channels with varying amplitude signals, in: Proc. IEEE Int. Symp. on Inform. Theory (ISIT), 1994, p. 381.

[9] B. Dulek, S. Gezici, Detector randomization and stochastic signaling for minimum probability of error receivers, IEEE Trans. Commun. 60 (4) (2012) 923928.

[10] C. Goken, S. Gezici, O. Arikan, Optimal stochastic signaling for power-constrained binary communications systems, IEEE Trans. Wirel. Commun. 9 (12) (2010) 3650-3661.

[11] C. Goken, S. Gezici, O. Arikan, On the optimality of stochastic signaling under an average power constraint, in: Proc. 48th Annual Allerton Conf. on Commun. Control, and Computing, Illinois, 2010, pp. 1158-1164.

[12] C. Goken, S. Gezici, O. Arikan, Optimal signaling and detector design for powerconstrained binary communications systems over non-Gaussian channels, IEEE Commun. Lett. 14 (2) (2010) 100-102.

[13] A. Patel, B. Kosko, Optimal noise benefits in Neyman-Pearson and inequalityconstrained signal detection, IEEE Trans. Signal Process. 57 (5) (2009) 16551669.

[14] E.G. Larsson, Improving the frame-error-rate of spatial multiplexing in block fading by randomly rotating the signal constellation, IEEE Commun. Lett. 8 (8) (2004) 514-516.

[15] E.G. Larsson, Constellation randomization (CoRa) for outage performance improvement on MIMO channels, in: IEEE Global Telecommunications Conference, vol. 1, 2004, pp. 386-390.

[16] Y. Li, C.N. Georghiades, G. Huang, Transmit diversity over quasi-static fading channels using multiple antennas and random signal mapping, IEEE Trans. Commun. 51 (11) (2003) 1918-1926.

[17] C. Lamy, J. Boutros, On random rotations diversity and minimum MSE decoding of lattices, IEEE Trans. Inf. Theory 46 (2000) 1584-1589.

[18] A. Hiroike, F. Adachi, N. Nakajima, Combined effects of phase sweeping transmitter diversity and channel coding, IEEE Trans. Veh. Technol. 41 (1992) 170176.

[19] X. Ma, G.B. Giannakis, Space-time-multipath coding using digital phase sweeping, in: IEEE Global Communications Conference, vol. 1, 2002, pp. 384-388.
[20] E.L. Lehmann, Testing Statistical Hypotheses, 2nd ed., Springer, 1997.

[21] K.E. Parsopoulos, M.N. Vrahatis, Particle swarm optimization method for constrained optimization problems, in: Intelligent Technologies-Theory and Applications: New Trends in Intelligent Technologies, IOS Press, 2002, pp. 214-220.

[22] S. Bayram, S. Gezici, H.V. Poor, Noise enhanced hypothesis-testing in the restricted Bayesian framework, IEEE Trans. Signal Process. 58 (8) (2010) 3972 3989.

[23] S. Bayram, S. Gezici, Noise-enhanced $M$-ary hypothesis-testing in the minimax framework, in: Proc. International Conference on Signal Processing and Commun. Systems, Omaha, NE, 2009, pp. 31-36.

[24] H. Soganci, S. Gezici, O. Arikan, Optimal stochastic parameter design for estimation problems, IEEE Trans. Signal Process. 60 (9) (2012) 4950-4956.

[25] S. Boyd, L. Vandenberghe, Convex Optimization, Cambridge University Press, Cambridge, UK, 2004.

[26] S. Shamai, S. Verdu, Worst-case power-constrained noise for binary-input channels, IEEE Trans. Inf. Theory 38 (1992) 1494-1511.

[27] J.G. Proakis, Digital Communications, 4th ed., McGraw-Hill, New York, 2001.

[28] A. Goldsmith, Wireless Communications, Cambridge University Press, Cambridge, UK, 2005.

[29] M.E. Tutay, S. Gezici, O. Arikan, Stochastic signal design on the downlink of a multiuser communications system, in: Proc. IEEE International Workshop on Signal Processing Advances for Wireless Communications (SPAWC), Cesme, Turkey, 2012.

[30] H. Chen, P.K. Varshney, S.M. Kay, J.H. Michels, Theory of the stochastic resonance effect in signal detection: Part I-Fixed detectors, IEEE Trans. Signal Process. 55 (7) (2007) 3172-3184.

[31] R.T. Rockafellar, R.J.-B. Wets, Variational Analysis, Springer-Verlag, Berlin, 2004.

[32] A. Maaref, S. Aissa, On the effects of Gaussian channel estimation errors on the capacity of adaptive transmission with space-time block coding, in: IEEE International Conference on Wireless and Mobile Computing, Networking and Communications (WiMob'2005), vol. 1, 2005, pp. 187-193.

[33] A. Ali Basri, T.J. Lim, Binary demodulation in Rayleigh fading with noisy channel estimates-Detector structures and performance, in: IEEE Vehicular Technology Conference (VTC Spring 2008), 2008, pp. 1162-1166.

[34] A. Vaz, E. Fernandes, Optimization of nonlinear constrained particle swarm, Balt. J. Sustain. 12 (1) (2006) 30-36.

Cagri Goken received the B.S. and M.S. degrees from Bilkent University, Ankara, Turkey in 2009 and 2011, respectively. He is currently working towards the Ph.D. degree in the Department of Electrical Engineering at Princeton University. His research interests are in the fields of information theory, wireless communications, and statistical signal processing.

Sinan Gezici received the B.S. degree from Bilkent University, Turkey in 2001, and the Ph.D. degree in Electrical Engineering from Princeton University in 2006. From 2006 to 2007, he worked at Mitsubishi Electric Research Laboratories, Cambridge, MA. Since February 2007, he has been an Assistant Professor in the Department of Electrical and Electronics Engineering at Bilkent University. Dr. Gezici's research interests are in the areas of detection and estimation theory, wireless communications, and localization systems. Among his publications in these areas is the book Ultra-wideband Positioning Systems: Theoretical Limits, Ranging Algorithms, and Protocols (Cambridge University Press, 2008).

Orhan Arikan was born in 1964 in Manisa, Turkey. In 1986, he received his B.Sc. degree in Electrical and Electronics Engineering from the Middle East Technical University, Ankara, Turkey. He received both his M.S. and Ph.D. degrees in Electrical and Computer Engineering from the University of Illinois, Urbana-Champaign, in 1988 and 1990, respectively. Following his graduate studies, he was employed as a Research Scientist at Schlumberger-Doll Research Center, Ridgefield, CT.

In 1993 he joined the Electrical and Electronics Engineering Department of Bilkent University, Ankara, Turkey. Since 2011, he is serving as the department chairman. His current research interests include statistical signal processing, time-frequency analysis and remote sensing. Dr. Arikan has served as Chairman of IEEE Signal Processing Society Turkey Chapter and President of IEEE Turkey Section.

\footnotetext{
6 When $x=0, G(x, \alpha)$ is independent of $\alpha$ and just a constant as it can be observed from (10)
} 